\title{
EFFECTIVE ELASTIC MODULI OF MISORIENTED SHORT-FIBER COMPOSITES
}

\author{
CHAO-HSUN CHEN and CHIANG-HO CHENG \\ Institute of Applied Mechanics, National Taiwan University, 1, Sec 4, Roosevelt Rd, \\ Taipei 106, Taiwan
}

(Received 19 November 1993; in revised form 7 July 1995)

\begin{abstract}
This paper deals with the effective moduli of composites containing misoriented fibers. The orientation distribution of fibers in a composite can be characterized by a single parameter exponential function, $F(\theta)=1-c^{-\lambda \theta}$. A large $\lambda$ indicates a highly oriented material, whereas a small $\lambda$ represents a quasi-isotropic material. Based on the Eshelby-Mori-Tanaka theory, the effective elastic moduli of planar orientation distribution and transversely isotropic distribution of fibers are studied. For planar orientation case, we have examined the nine independent stiffness constants of injection-molded tensile bars of poly (pheylene sulfide) reinforced with 30 and $40 \%$ by weight of carbon or glass fibers which have been measured by Choy et al. [Polymer Composites 13, 69-80 (1992)]. For the transversely isotropic orientation case, five elastic constants of a composite are derived. These moduli are given in terms of the length-diameter aspect ratio, the volume fraction, and the orientation distribution parameter $\lambda$ of the short fibers. And they are given in very simple, explicit forms. Numerical results are presented to demonstrate the effects of the fiber elastic property, aspect ratio, volume fraction and orientation distribution function on composite elastic moduli. The fiber orientation distribution and aspect ratio have a more significant effect on composite longitudinal Young's modulus than does the fiber volume fraction within the range examined. Copyright (C) 1996 Elsevier Science Ltd.
\end{abstract}

\section{INTRODUCTION}

Recently, thermoplastics reinforced by short glass or carbon fibers have received considerable attention because they can be easily produced by low-cost injection molding or extrusion compounding processes.

To predict the elastic modulus of a fiber-reinforced thermoplastic, the simulated laminated-plate model is often used (Kacir et al., 1975; Tsai and Hahan, 1980). FRTP is treated as unidirectional plies or laminae stacked up in a particular arrangement of orientation. The first thorough study of injection-molded short-carbon-fiber-reinforced thermoplastics was undertaken by Hahn et al. (1986). They measured the stiffness in the axial, transverse, and thickness directions of ASTM type 1 tensile bars by an ultrasonic method and converted these to the respective Young's moduli using theoretical modulus to stiffness ratios. The degree of fiber orientation is expected to be higher in the skin layer, so to study this effect, measurements were made by Choy et al. (1992) in both the skin and core regions. They have measured the nine independent elastic moduli and three principal thermal conductivities of injection-molded tensile bars of PPS reinforced with 30 and 40 weight $\%$ of carbon or glass fibers. The distributions of the aspect ratio and orientation of the fibers were also determined. Using these data, the elastic moduli were calculated via the laminate theory. The above discussion points to the fact that there is a $10 \%$ difference between the theoretical predictions and experimental results. The elastic moduli predicted based on the laminate theory has the limintation of its laminate-plate-theory assumption. An orientation distribution generally requires a three-dimensional description. However, if a thin sheet specimen has a geometry such that the thickness dimension is much less than the width and length dimensions, then a simplified planar orientation of fibers can be assumed or if a specimen has a geometry such that the fiber distribution function is axisymmetric with respect to the major axis of the specimen, say the $x_{1}$ axis, then the material as a whole possesses transversely isotropic properties in the 2-3 plane. Kacir et al. (1975) proposed a distribution function representing this planar orientation which has the property that 
variation of its function's shape is able to describe a change from a unidirectional distribution to a random planar distribution.

For short-fiber reinforced thermoplastics (FRTP), the two most important microstructural parameters are fiber length and fiber orientation. The case of three-dimensional misaligned fiber composites has been analysed (Christensen, 1979; Chou and Nomura, 1980). In their analyses, the interactions between fibers at different orientations were not considered. Chou and Nomura (1980) used the results from both the bound and selfconsistent approaches (Chou et al., 1980) for the values for each orientation. Takao et al. (1982) considered the interaction among fibers at different orientations by adopting the average induced strain approach (Taya and Chou, 1981; Taya and Mura, 1981) and the modified Eshelby's equivalent inclusion method (Eshelby, 1957). In this paper, we consider the effect of the distribution in fiber orientation on the effective elastic moduli. The interaction among fibers at different orientations is included in the analysis by adopting the mean stress concept of Mori and Tanaka (1973) and putting it together with the eigenstrain idea of Eshelby (1957) to predict the effective moduli of FRTP. The orientation distribution of fibers in a composite is characterized by Kacir's single parameter exponential function. These moduli are given in terms of the length-diameter aspect ratio, the volume fraction, and the orientation distribution parameter of the short fibers.

\section{EFFECTIVE ELASTIC MODULI}

If an infinite elastic body (without inclusions, i.e., the matrix only) subjected to a uniform stress field, $\sigma_{0}$. The uniform strain, $\varepsilon_{0}$, produced is

$$
\varepsilon_{0}=\mathbf{C}_{m}^{-1} \cdot \boldsymbol{\sigma}_{0}
$$

in which $\mathbf{C}_{m}$ is the elastic modulus tensor of the matrix (which is assumed to be isotropic).

When there are ellipsoidal inclusions (fibers) present in the matrix, a perturbed stress field is induced and is denoted as $\tilde{\sigma}(x)$.

The total stress field, $\sigma(x)$, is now the sum of two stress fields: $\sigma_{0}+\tilde{\sigma}(x)$. Define the volumetric average of the perturbed stress and strain fields of the matrix, $\bar{\sigma}$ and $\bar{\varepsilon}$, according to

$$
\overline{\boldsymbol{\sigma}}=\langle\tilde{\boldsymbol{\sigma}}(x)\rangle_{D-\Omega}=\frac{1}{V_{D-\Omega}} \int_{D-\Omega} \tilde{\boldsymbol{\sigma}}(x) \mathrm{d} V=\mathbf{C}_{m} \cdot \overline{\boldsymbol{\varepsilon}}
$$

in which $D, \Omega, D-\Omega$ are the domain of the whole elastic body (composite), all the fibers and the matrix, respectively, $V_{D-\Omega}$ is the volume of the matrix, and \langle\rangle$_{D-\Omega}$ is the volumetric average for the domain $D-\Omega$. Thus, the average stress field in the matrix is

$$
\boldsymbol{\sigma}_{m}=\boldsymbol{\sigma}_{0}+\overline{\boldsymbol{\sigma}}=\mathbf{C}_{m} \cdot\left(\varepsilon_{0}+\overline{\boldsymbol{\varepsilon}}\right)
$$

if the composite contains a single phase of ellipsoidal inhomogeneities. When all inhomogeneities are aligned in one direction, the following two equations (Taya and Mura, 1981) are obtained :

$$
\mathbf{C}_{m}\left[\bar{\varepsilon}+f_{1}\left(\varepsilon^{\mathrm{pl}}-\boldsymbol{\varepsilon}^{*}\right)\right]=0,
$$

denote $\mathbf{C}_{f}$ as the elastic modulus tensor of the fiber :

$$
\begin{aligned}
\sigma_{f} & =\mathbf{C}_{f} \cdot \boldsymbol{\varepsilon}_{f}=\mathbf{C}_{f} \cdot\left(\varepsilon_{0}+\bar{\varepsilon}+\varepsilon^{\mathrm{pt}}\right) \\
& =\mathbf{C}_{m} \cdot\left(\varepsilon_{0}+\bar{\varepsilon}+\varepsilon^{\mathrm{pt}}-\boldsymbol{\varepsilon}^{*}\right),
\end{aligned}
$$


where $\varepsilon^{*}$ is Eshelby's (1957) equivalent transformation strain [or Mura's eigenstrain (1982)]. The perturbed strain, $\varepsilon^{\mathrm{pt}}$, is taken to be related to $\varepsilon^{*}$ as

$$
\varepsilon^{\mathrm{pt}}=\mathbf{S} \cdot \boldsymbol{\varepsilon}^{*}
$$

in which $\mathbf{S}$ is the Eshelby tensor associated with spheroidal inclusions and $\mathbf{C}_{m}$ (Eshelby, 1957; Mura, 1982).

Furthermore, the total volumetric average strain of the composite material $\boldsymbol{\varepsilon}^{\mathrm{T}}$ can be represented as

$$
\boldsymbol{\varepsilon}^{\mathrm{T}}=f_{0}\left(\varepsilon_{0}+\bar{\varepsilon}\right)+f_{1}\left(\varepsilon_{0}+\bar{\varepsilon}+\varepsilon^{\mathrm{pt}}\right)=\varepsilon_{0}+f_{1} \varepsilon^{*}
$$

The result of eqn (7) is derived by using eqn (4). From eqns (5) and (6), we have

$$
\varepsilon^{*}=\mathbf{A} \cdot \mathbf{T} \cdot\left(\varepsilon_{0}+\bar{\varepsilon}\right)=\mathbf{B} \cdot\left(\varepsilon_{0}+\bar{\varepsilon}\right),
$$

in which $\mathbf{A}, \mathbf{T}$, and $\mathbf{B}$ can be represented as

$$
\begin{gathered}
\mathbf{B}=\mathbf{A} \cdot \mathbf{T} \\
\mathbf{A}=\mathbf{I}-\mathbf{C}_{m}^{-1} \cdot \mathbf{C}_{f} \\
\mathbf{T}=\left(\mathbf{I}+\mathbf{S} \cdot \mathbf{C}_{m}^{-1} \cdot \mathbf{C}_{f}-\mathbf{S}\right)^{-1},
\end{gathered}
$$

and $\mathbf{I}$ is the fourth-order tensor, which can be defined as

$$
\mathbf{I}_{p q r s}=\frac{1}{2}\left(\delta_{p r} \delta_{q s}+\delta_{p s} \delta_{q r}\right)
$$

Now from eqn (6), we have

$$
\boldsymbol{\varepsilon}^{\mathrm{pt}}-\boldsymbol{\varepsilon}^{*}=(\mathbf{S}-\mathbf{I}) \cdot \mathbf{A} \cdot \mathbf{T} \cdot\left(\boldsymbol{\varepsilon}_{0}+\overline{\boldsymbol{\varepsilon}}\right)=\mathbf{E} \cdot\left(\boldsymbol{\varepsilon}_{0}+\overline{\boldsymbol{\varepsilon}}\right),
$$

where

$$
\mathbf{E}=(\mathbf{S}-\mathbf{I}) \cdot \mathbf{A} \cdot \mathbf{T}
$$

Substituting eqn (10) into eqn (4) leads to

$$
\overline{\mathbf{s}}+f_{1} \mathbf{E} \cdot\left(\varepsilon_{0}+\bar{\varepsilon}\right)=0 .
$$

Rearranging eqn (12), we have

$$
\varepsilon_{0}+\overline{\boldsymbol{\varepsilon}}=\left(I+f_{1} \mathbf{E}\right)^{-1} \cdot \varepsilon_{0}
$$

Substituting eqn (13) into eqn (7) leads to

$$
\boldsymbol{\varepsilon}^{\mathrm{T}}=\left[\mathbf{I}+f_{1} \mathbf{B} \cdot\left(\mathbf{I}+f_{1} \mathbf{E}\right)^{-1}\right] \cdot \boldsymbol{\varepsilon}_{0} .
$$

The effective moduli, $\mathbf{C}^{*}$, can be defined based on the following relation

$$
\boldsymbol{\sigma}_{0}=\mathbf{C}^{*} \cdot \boldsymbol{\varepsilon}^{\mathrm{T}}
$$

The effective elastic moduli, $\mathbf{C}^{*}$, then can be determined from eqns (1), (14) and (15) as follows: 


$$
\mathbf{C}^{*}=\mathbf{C}_{m}\left[\mathbf{I}+f_{1} \mathbf{B} \cdot\left(I+f_{1} \mathbf{E}\right)^{-1}\right]^{-1},
$$

Consider the distribution of fiber orientation. The coordinate system is defined so that the fibers are in the 1-2 plane with the orientation angle $\theta$ being measured relative to the 1 axis. Let $x_{i}^{\prime}$ be the local coordinate system of the inclusion, and $x_{i}$ be the global coordinate system of whole elastic body (composite material). By using the spherical coordinate system, their relation then can be represented as

$$
x^{\prime}=Q \cdot x
$$

where

$$
Q_{i j}=\left[\begin{array}{ccc}
\cos \theta & \sin \theta \cos \phi & \sin \theta \sin \phi \\
-\sin \theta & \cos \theta \cos \phi & \cos \theta \sin \phi \\
0 & -\sin \phi & \cos \phi
\end{array}\right]
$$

Since $\mathbf{B}$ and $\mathbf{E}$ in eqn (16), they are both fourth-order tensor, after being transformed into the global coordinate system then can be written as a representative form

$$
A_{i j k l}^{\prime}=Q_{i p} Q_{j q} Q_{k r} Q_{l s} A_{p q r s} .
$$

The general expression of the volumetric average after arbitrary material forming history becomes

$$
\bar{A}_{i j k i}=\frac{1}{V} \int_{\phi}^{\phi} \int_{-i H^{\prime}}^{\theta t} A_{i j k}^{\prime} f(\theta) \mathrm{d} \theta \mathrm{d} \phi
$$

where $\phi^{\prime}$ and $\theta^{\prime}$ are the ranges of integration in the $\phi$ and $\theta$ direction, respectively, and $V$ is the volume of relevant segment of a sphere with unit radius.

Therefore, the general expression for the effective elastic moduli, $\mathbf{C}^{*}$, after any material forming process described above can be calculated by assuming statistical homogeneity and integrating the stiffnesses determined by substuting the relation of eqn (20) into eqn (16) as follows

$$
\mathbf{C}^{*}=\mathbf{C}_{m}\left[\mathbf{I}+f_{1} \overline{\mathbf{B}} \cdot\left(I+f_{1} \overline{\mathbf{E}}\right)^{-1}\right]^{-1}
$$

where

$$
\overline{\mathbf{B}}=\langle B\rangle \quad \text { and } \quad \overline{\mathbf{E}}=\langle E\rangle .
$$

\section{FIBER ORIENTATION DISTRIBUTION}

Any material forming processes, such as injection, extrusion, and rolling of an initially isotropic material, causes a permanent fiber orientation which changes the material properties from isotropic to either transversely isotropic or orthotropic. The changes are related in a complex way to the geometry of the fibers, melt behavior of the polymer matrix, mold cavity and the filling process. An orientation generally requires a three-dimensional description.

If a symmetrical angular fiber distribution function around the major alignment direction is assumed, the experimental fiber distribution data can be used to build a complex symmetrical laminate of weighted groups of angle plies oriented $\pm \theta$ degrees from the major alignment axis. With this assumption, Kacir et al. (1975), proposed a single parameter exponential function to describe the fiber orientation distribution in a molded specimen. Definition of Kacir's orientation distribution function is as follows : 


$$
\begin{aligned}
& \rho(\theta)=\lambda \mathrm{e}^{-\lambda \theta} \\
& F(\theta)=\int_{0}^{\theta} \lambda \mathrm{e}^{-\lambda x} \mathrm{~d} x=1-\mathrm{e}^{-\lambda \theta}
\end{aligned}
$$

where $F(\theta)$ is the accumulated percent of fiber oriented between 0 to $\pm \theta, \rho(\theta)$ is the density function of fibers oriented at $\pm \theta$; $\lambda$ is the shape parameter; and $\theta$ is the orientation angle ranging from 0 to $\pi / 2$. Large $\lambda$ indicates that fibers have a major preferential alignment. As $\lambda$ decreases, the orientation of fibers in the sample will become more random.

\subsection{Planar orientation of fibers}

However, if a thin sheet specimen has a geometry such that its thickness is much less than its width and length, then a simplified planar orientation of fibers can be assumed. Thus eqn (21) can be defined as follows :

$$
\bar{A}=\left\langle A\left(\theta, \phi_{0}\right) \geqslant \frac{\int_{-\pi / 2}^{0} A^{\prime}\left(\theta, \phi_{0}\right) \rho(-\theta) \mathrm{d} \theta+\int_{0}^{\pi / 2} A^{\prime}\left(\theta, \phi_{0}\right) \rho(\theta) \mathrm{d} \theta}{2 \int_{0}^{\pi / 2} \rho(\theta) \mathrm{d} \theta} .\right.
$$

\subsection{Transverse isotropy in the 2-3 plane}

In this section we consider the case where the distribution function $\rho(\theta, \phi)$ is axisymmetric with respect to the major axis of the specimen, say $x_{1}$ axis, so that the material as a whole possesses transversely isotropic properties in the $2-3$ plane. It means that $\rho$ is a function of $\theta$ only. Thus, eqn (21) can be defined as follows:

$$
\bar{A}=\left\langle A(\theta, \phi) \geqslant \frac{\int_{0}^{2 \pi} \int_{0}^{\pi / 2} A^{\prime}(\theta, \phi) \rho(\theta) \sin \theta \mathrm{d} \theta \mathrm{d} \phi}{\int_{0}^{2 \pi} \int_{0}^{\pi / 2} \rho(\theta) \sin \theta \mathrm{d} \theta \mathrm{d} \phi} .\right.
$$

\section{RESULTS AND DISCUSSION}

\subsection{Elastic moduli of the matrix and fibers}

The elastic moduli of a short fiber-reinforced composite depend on the elastic moduli of the fiber and matrix, and the volume fraction $f_{1}$, aspect ratio, $l / d$, and degree of orientation (characterized by the parameter $\lambda$ ) of the fibers. Their values are shown in Table 1 [from Choy et al. (1992)].

Table 1. The elastic properties and density of carbon and glass fibers and PPs matrix

\begin{tabular}{lccc}
\hline & $\begin{array}{c}\text { Carbon fiber } \\
\text { (Thronel 300) }\end{array}$ & E glass fiber & PPS matrix \\
\hline$E_{11}(\mathrm{Gpa})$ & 231 & 76 & 4 \\
$E_{22}(\mathrm{Gpa})$ & 13.8 & 76 & 4 \\
$G_{23}(\mathrm{Gpa})$ & 5.52 & 30.4 & 1.43 \\
$G_{12}(\mathrm{Gpa})$ & 12.4 & 30.4 & 1.43 \\
$v_{12}$ & 0.2 & 0.25 & 0.4 \\
$v_{23}$ & 0.25 & 0.25 & 0.4 \\
$\rho\left(\mathrm{g} / \mathrm{cm}^{3}\right)$ & 1.75 & 2.56 & 1.36 \\
\hline
\end{tabular}


The fibers have a much higher modulus than the matrix, and this would lead to stiffness enhancement in composites. The effect is expected to be much stronger in carbon-fiberreinforced composites since $E_{11}^{f} / E_{11}^{m}=58$ for carbon fibers compared with 19 for glass fibers.

\subsection{Elastic moduli of injection-molded short-fiber reinforced thermoplastics}

4.2.1. Fiber orientation and aspect ratio. In Section 3.1, if a thin shect specimen has a geometry such that the thickness dimension is much less than the width and length dimensions, then a simplified planar orientation of fibers can be assumed. We have employed the experimental results of Choy et al. (1992) for the orientation of fibers in the surface layer (at a depth of $0.3 \mathrm{~mm}$ ) and middle layer (at a depth of $1.6 \mathrm{~mm}$ ) of three composites: PPS30cf, PPS 40cf, and PPS 40gf as follows:

(1) The fibers in the surface layer are aligned close to the mold flow direction ( $1^{\prime}$ axis), making an average angle $\langle\theta\rangle$ of about $15^{\circ}$ with this axis. Moreover, the fiber orientation is roughly the same at all positions along the width.

(2) The fiber orientation is also very high near the two edges (width positions 0.5 and 12 $\mathrm{mm}$ ) of the middle layer, but becomes increasingly random as one moves toward the core (width position 4-8 $\mathrm{mm}$ ).

(3) The fiber orientation distribution for a PPS30cf sample trimmed to a width of $8 \mathrm{~mm}$ ( $\pm 4 \mathrm{~mm}$ from central line) showed that more than $60 \%$ of the fibers in the surface layer lie at an angle of less than 15 relative to the $1^{\prime}$ axis, and there are almost no fibers in the range of $6090^{\circ}$. The middle layer exhibits a broader angular distribution, with a significant number of fibers lying at large angles.

(4) The fibers in each composite have a wide range of aspect ratios. However, the average aspect ratio falls within the narrow range of 16-21 for all three composites.

From these data, the cumulative fiber distribution $F(\theta)$, i.e., the fraction of fibers oriented at an angles smaller than $\theta$, was calculated and by fitting the $F(\theta)$ data to the expression in eqn (23), the orientation factors $\lambda$ for the three composites were obtained (Table 2). It should be noted that $\lambda=0$ and $\lambda=\infty$ correspond to random and perfect orientation, respectively, and so the value of $\lambda$ provides a quantitative measure of the degree of fiber alignment. All three composites have similar $\lambda$ values, with $\lambda=4.1-5.3$ in the surface layer and $1.9-2.9$ in the middle layer.

4.2.2. Elastic moduli of the composites. The nine independent stiffnesses, $C_{i j}^{*}$, and three Young's moduli, $E_{11}, E_{22}$ and $E_{33}$ of the composites are given in Table 3. Since the tensile and shear moduli are of greater theoretical interest and practical importance, we will concentrate our attention on these parameters. Compared with the tensile and shear moduli of $E_{11}^{m}=4 \mathrm{GPa}$ and $G_{12}^{m}=1.43 \mathrm{GPa}$ for the PPS matrix, the elastic moduli of the composites are significantly higher, clearly revealing the reinforcing effect of the fibers. The longitudinal Young's modulus $E_{11}$ and longitudinal shear modulus $C_{66}\left(\equiv G_{12}\right)$ show that the greatest increase which results from the fact the fibers lie in the $1^{\prime}-2^{\prime}$ plane with preferential alignment along the $1^{\prime}$ axis. Since the surface layer has a higher degree of fiber orientation than the middle layer it has a higher $E_{11}$ but lower $E_{22}, E_{33}$, and $G_{12}$ (skin-core effect). The

Table 2. The volume fraction, $f_{1}$, averaged aspect ratio, $l / d$, and orientation factor, $i$ of the fibers in three composites

\begin{tabular}{lcccc}
\hline & $f_{1}$ & $\begin{array}{c}\text { Average aspect } \\
\text { ratio }\end{array}$ & Surface layer & Middle layer \\
\hline PPS30cf & 0.24 & 21 & 4.1 & 1.9 \\
PPS40cf & 0.34 & 16 & 4.7 & 2.1 \\
PPS40gf & 0.26 & 17 & 5.3 & 2.9 \\
\hline
\end{tabular}


Table 3. The stiffness and Young's moduli (in unit of Gpa) of three composites

\begin{tabular}{|c|c|c|c|c|c|c|}
\hline & \multicolumn{2}{|c|}{ PPS30cf $(24.3 \%)$} & \multicolumn{2}{|c|}{ PPS40cf $(33.5 \%)$} & \multicolumn{2}{|c|}{ PPS40gf $(26.4 \%)$} \\
\hline & Surface & Middle & Surface & Middle & Surface & Middle \\
\hline$\lambda$ & 4.1 & 1.9 & 4.7 & 2.1 & 5.3 & 2.9 \\
\hline$C_{11}^{*}$ & $\begin{array}{c}36.96 \\
(38.0)\end{array}$ & $\begin{array}{c}31.04 \\
(31.9)\end{array}$ & $\begin{array}{r}44.11 \\
(41.7)\end{array}$ & $\begin{array}{c}36.7 \\
(36.0)\end{array}$ & $\begin{array}{r}23.02 \\
(24.7)\end{array}$ & $\begin{array}{r}21.66 \\
(21.2)\end{array}$ \\
\hline$C_{22}^{*}$ & $\begin{array}{c}10.56 \\
(10.0)\end{array}$ & $\begin{array}{c}13.86 \\
(12.5)\end{array}$ & $\begin{array}{c}10.76 \\
(10.8)\end{array}$ & $\begin{array}{c}14.43 \\
(15.1)\end{array}$ & $\begin{array}{r}12.11 \\
(12.1)\end{array}$ & $\begin{array}{r}12.91 \\
(13.0)\end{array}$ \\
\hline$C_{33}^{*}$ & $\begin{array}{c}9.44 \\
(9.50)\end{array}$ & $\begin{array}{r}9.44 \\
(10.5)\end{array}$ & $\begin{array}{r}9.83 \\
(10.3)\end{array}$ & $\begin{array}{r}9.83 \\
(10.9)\end{array}$ & $\begin{array}{r}11.88 \\
(11.5)\end{array}$ & $\begin{array}{r}11.88 \\
(12.0)\end{array}$ \\
\hline$C_{44}^{*}$ & $\begin{array}{c}1.89 \\
(1.84)\end{array}$ & $\begin{array}{c}1.93 \\
(2.74)\end{array}$ & $\begin{array}{c}2.11 \\
(1.99)\end{array}$ & $\begin{array}{c}2.16 \\
(2.78)\end{array}$ & $\begin{array}{c}2.23 \\
(2.40)\end{array}$ & $\begin{array}{c}2.24 \\
(2.65)\end{array}$ \\
\hline$C_{55}^{*}$ & $\begin{array}{c}2.09 \\
(2.28)\end{array}$ & $\begin{array}{c}2.06 \\
(3.09)\end{array}$ & $\begin{array}{c}2.44 \\
(2.55)\end{array}$ & $\begin{array}{c}2.38 \\
(3.28)\end{array}$ & $\begin{array}{c}2.34 \\
(2.52)\end{array}$ & $\begin{array}{c}2.33 \\
(2.95)\end{array}$ \\
\hline$C_{66}^{*}$ & $\begin{array}{c}4.20 \\
(3.42)\end{array}$ & $\begin{array}{c}5.56 \\
(5.30)\end{array}$ & $\begin{array}{c}4.62 \\
(3.86)\end{array}$ & $\begin{array}{c}6.42 \\
(5.80)\end{array}$ & $\begin{array}{c}3.02 \\
(3.12)\end{array}$ & $\begin{array}{c}3.54 \\
(3.45)\end{array}$ \\
\hline$C_{23}^{*}$ & $\begin{array}{c}5.63 \\
(5.20)\end{array}$ & $\begin{array}{c}5.45 \\
(5.50)\end{array}$ & $\begin{array}{c}5.58 \\
(6.10)\end{array}$ & $\begin{array}{c}5.31 \\
(6.30)\end{array}$ & $\begin{array}{c}7.42 \\
(7.10)\end{array}$ & $\begin{array}{c}7.38 \\
(7.10)\end{array}$ \\
\hline$C_{13}^{*}$ & $\begin{array}{c}5.38 \\
(5.20)\end{array}$ & $\begin{array}{c}5.22 \\
(5.60)\end{array}$ & $\begin{array}{c}5.32 \\
(6.20)\end{array}$ & $\begin{array}{c}5.00 \\
(6.40)\end{array}$ & $\begin{array}{c}7.07 \\
(7.10)\end{array}$ & $\begin{array}{c}7.10 \\
(6.90)\end{array}$ \\
\hline$C_{12}^{*}$ & $\begin{array}{c}7.16 \\
(6.20)\end{array}$ & $\begin{array}{c}8.29 \\
(5.90)\end{array}$ & $\begin{array}{c}7.06 \\
(7.20)\end{array}$ & $\begin{array}{c}8.37 \\
(6.70)\end{array}$ & $\begin{array}{c}7.64 \\
(7.50)\end{array}$ & $\begin{array}{c}8.14 \\
(7.20)\end{array}$ \\
\hline$E_{1 !}$ & $\begin{array}{c}31.50 \\
(33.6)\end{array}$ & $\begin{array}{c}25.3 \\
(28.1)\end{array}$ & $\begin{array}{c}38.76 \\
(36.2)\end{array}$ & $\begin{array}{r}30.96 \\
(31.5)\end{array}$ & $\begin{array}{c}17.42 \\
(19.2)\end{array}$ & $\begin{array}{r}15.74 \\
(16.1)\end{array}$ \\
\hline$E_{22}$ & $\begin{array}{c}6.68 \\
(6.80)\end{array}$ & $\begin{array}{c}9.59 \\
(9.30)\end{array}$ & $\begin{array}{c}7.12 \\
(6.90)\end{array}$ & $\begin{array}{c}10.4 \\
(11.2)\end{array}$ & $\begin{array}{c}6.93 \\
(7.20)\end{array}$ & $\begin{array}{c}7.52 \\
(8.20)\end{array}$ \\
\hline$E_{33}$ & $\begin{array}{c}6.33 \\
(6.70)\end{array}$ & $\begin{array}{c}7.05 \\
(7.80)\end{array}$ & $\begin{array}{c}6.82 \\
(6.70)\end{array}$ & $\begin{array}{c}7.61 \\
(7.90)\end{array}$ & $\begin{array}{c}7.02 \\
(7.00)\end{array}$ & $\begin{array}{c}7.30 \\
(7.60)\end{array}$ \\
\hline
\end{tabular}

Note: ( ) represents the experimental results of Cho et al. (1992)

alignment of fibers also leads to strong anisotropy in the elastic properties. $E_{11}$ for carbonfiber composites is substantially higher than $E_{22}$, which in turn, is slightly larger than $E_{33}$. Similarly, $C_{66}>C_{55}>C_{44}$. These anisotropies reflect the high anisotropy of the fiber. Since glass fiber is isotropic and has a lower modulus than carbon fiber, $E_{11}$ for the glass-fiber composite at a similar $f_{1}$ is lower than that of carbon-fiber composite by a factor of about 2 [the $E_{11}$ values of PPS30cf $\left(f_{1}=0.24\right)$ and PPS $40 \mathrm{gf}\left(f_{1}=0.26\right)$ in Table 3 may be compared].

Besides fiber orientation, there are two other factors that influence the elastic moduli: fiber volume fraction $f_{1}$, and aspect ratio $l / d$. Table 3 shows that for glass-fiber composites, $E_{11}$ is roughly proportional to $f_{1}$, but the dependence is weaker for other moduli. However, $E_{11}$ for PPS40cf is only $10 \%$ higher than that for PPS30cf although $f_{1}$ is more than $30 \%$ higher. The small difference in $E_{11}$ arises largely from the fact that PPS40cf has a much smaller aspect ratio (see Table 2). For the three composite samples, PPS30cf, PPS40cf, and PPS40gf, all the relevant parameters have been determined, and the elastic moduli can be calculated according to the Mori-Tanaka mean field theory in conjunction with Eshelby's equivalent-inclusion method discussed earlier (see Section 1). As shown in Table 4 the theoretical predictions and experimental results differ by less than $10 \%$ on average.

The good agreement implies that the structural model we have used to describe injection-molded composites is reasonable and that the theory provides a satisfactory theoretical framework.

To gain a deeper understanding of the effects of various parameters, we have calculated and plotted the elastic moduli as functions of the aspect ratio, $l / d$. Figure 1 shows that all the moduli for carbon-fiber composites increase with increasing $l / d$, but the increase is much more significant for the longitudinal moduli $E_{11}$ and $G_{12}$. In the $l / d$ range of $10-20, E_{11}$ for 
Table 4. Comparison of the experimental and theoretical values of the elastic moduli (in unit Gpa) of three composites

\begin{tabular}{lcccc}
\hline & & \multicolumn{3}{c}{ Deviation (\%) } \\
\cline { 3 - 4 } & & PPS30cf & PPS40cf & PPS40gf \\
\hline Surface layer & $E_{11}$ & 6.67 & -6.6 & 10.22 \\
& $E_{22}$ & 1.8 & -3.09 & 3.9 \\
& $E_{33}$ & 5.85 & -1.75 & 0 \\
Middle layer & $G_{12}$ & -18.57 & -16.4 & 3.3 \\
& $E_{11}$ & 11.07 & 1.74 & 2.29 \\
& $E_{22}$ & 3.02 & 7.7 & 9.04 \\
& $E_{33}$ & 10.64 & 3.81 & -2.54 \\
\hline
\end{tabular}

(a)

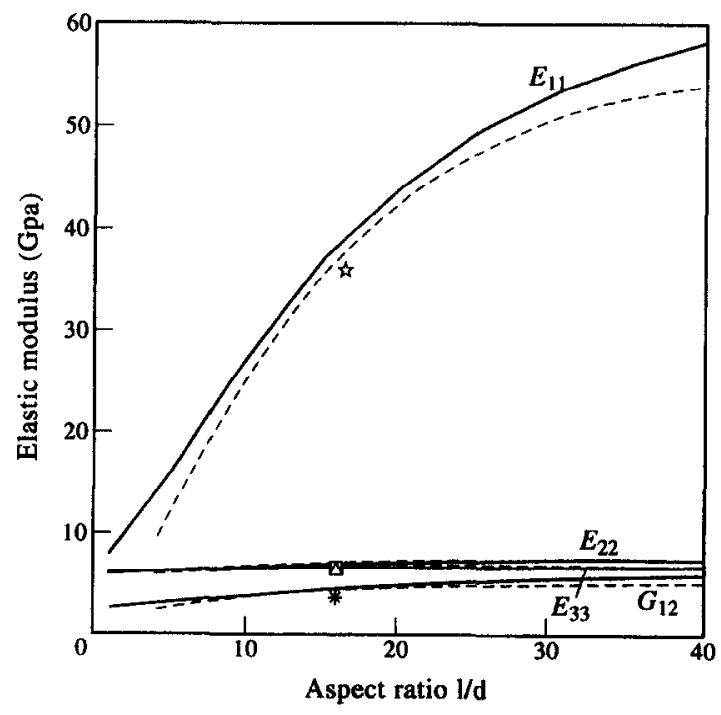

(b)

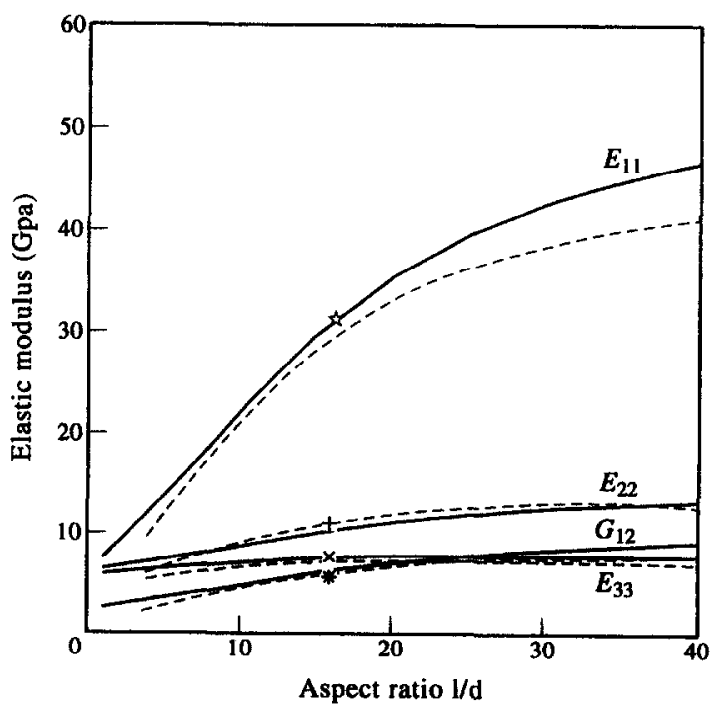

Fig. 1. Elastic moduli of 40 weight \% carbon-fiber-rcinforced PPS as functions of fiber aspect ratio (a) surface layer, (b) middle layer. Symbols represent the experimental data. The theoretical curves are calculated by taking $\lambda=4.7$ and 2.1 in (a) and (b), respectively: solid line--present model dashed line-laminated plate model. 
(a)

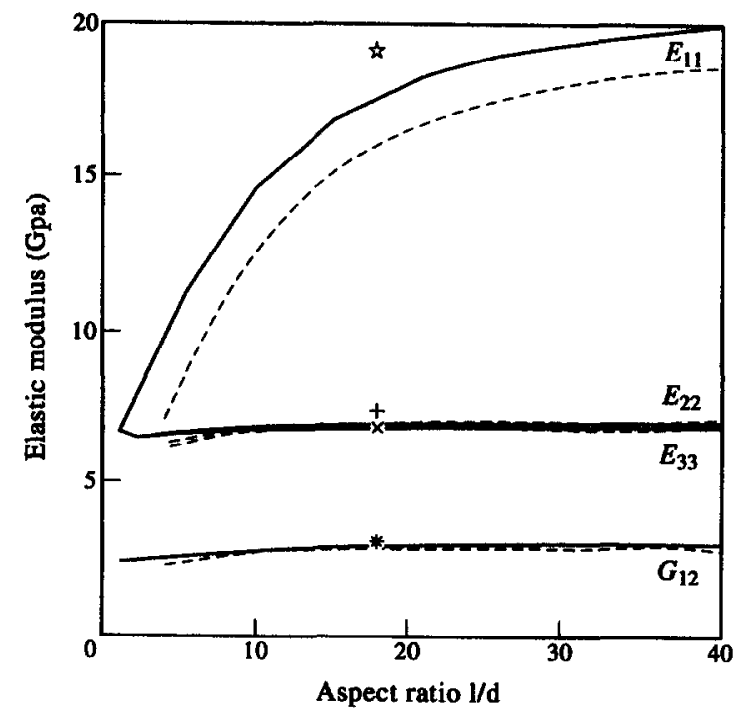

(b)

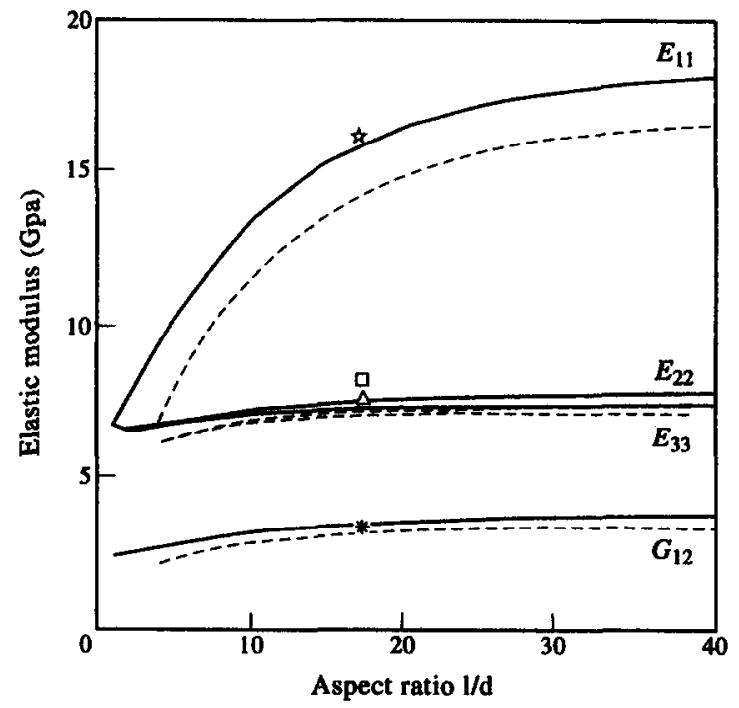

Fig. 2. Elastic moduli of 40 weight $\%$ glass-fiber-reinforced PPS as functions of fiber aspect ratio: (a) surface layer, (b) middle layer. Symbols represent the experimental data. The theoretical curves are calculated by taking $\lambda=5.3$ and 2.9 in (a) and (b), respectively: solid line present model; dashed line-laminated plate model.

both layers and $G_{12}$ for the middle layer increase almost linearly with $l / d$. These results emphasize the importance of choosing a suitable processing technique to increase the fiber aspect ratio. The situation is somewhat different for glass-fiber-reinforced composites (Fig. 2). Although $E_{11}$ also increases sharply at low aspect ratios, the effect becomes saturated above $l / d=15$. This arises from the fact that $E_{11}$ of glass fibers is much smaller than that of carbon fibers.

Next we turn our attention to the dependence on fiber volume fraction $f_{1}$ (Figs 3 and $4)$. In the typical $f_{1}$ range of $0.2-0.35$ for short-carbon-fiber composites, $E_{11}$ increases slightly faster than linearly with $f_{1}$ at all fiber orientations. However, the slope of the curve becomes smaller than as $\lambda$ decreases. At $f_{1}=0.335$ (the fiber volume fraction of PPS40cf), $E_{11}$ is higher than the matrix modulus $E_{11}^{m}$ by a factor of 4.5 for fibers randomly oriented 
(a)

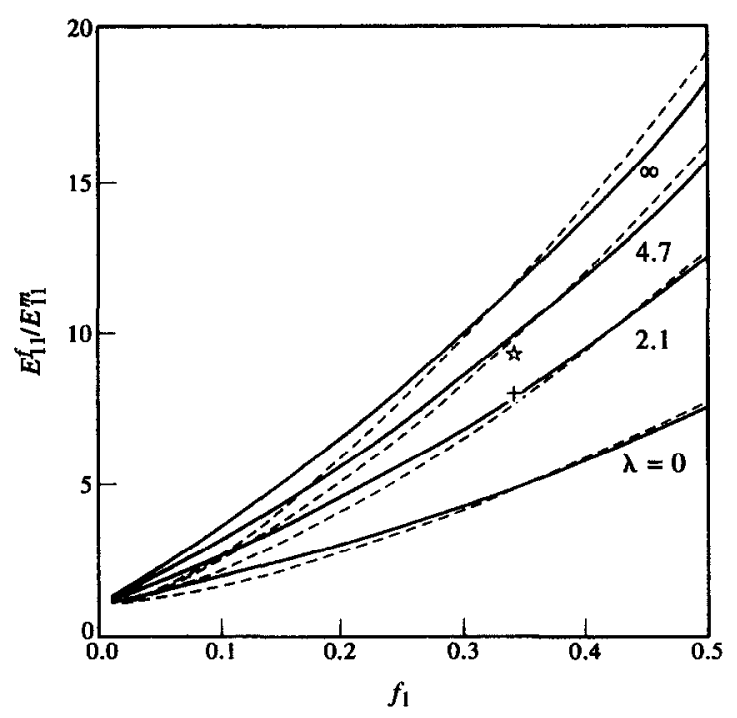

(b)

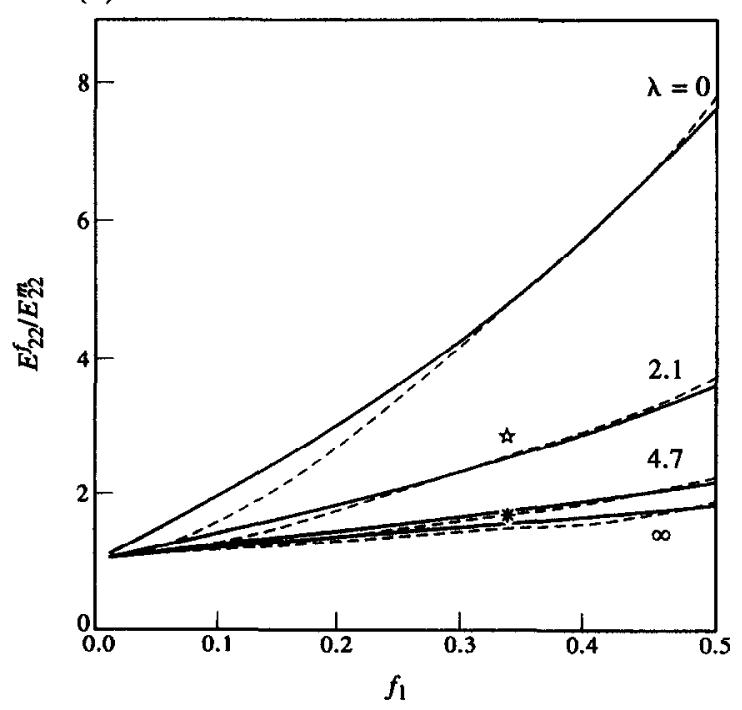

Fig. 3. Relative tensile modulus of carbon-fiber-reinforced PPS as functions of fiber volume fraction $f_{1}$. The solid and dashed curves are the theoretical predictions for: (a) $E_{11}$ and (b) $E_{22}$, respectively, taking $t=16$. Symbols represent the experimental data : solid line-present model ; dashed linelaminated plate model.

in the $1^{\prime}-2^{\prime}$ plane $(\lambda=0)$ and 1 for fibers aligned in the $1^{\prime}$ direction $(\lambda=\infty)$. This is the range of enhancement we can expect for the tensile modulus along the flow direction of injection-molded bars. The orientation factor $(\lambda=4.7)$ of the surface layer of PPS40cf is sufficiently high that its $E_{11}^{f} / E_{11}^{m}$ value of 9 is smaller than that for fully aligned fibers by only $20 \%$. At this orientation, $E_{11}$ has half the value of a composite with aligned continuous fibers (Fig. 3). Contrary to the behavior of $E_{11}$, the slopes of the curves for $E_{22}$ and $G_{12}$ increases as the fiber orientation becomes random.

\subsection{Effective moduli for transversely isotropic composites}

4.3.1. Explicit expression of the effective moduli. In Section 3.2, if $\mathbf{C}(\theta, \phi)$ is the axisymmetric orientation with respect to the major axis of the specimen as in eqn (25), then 


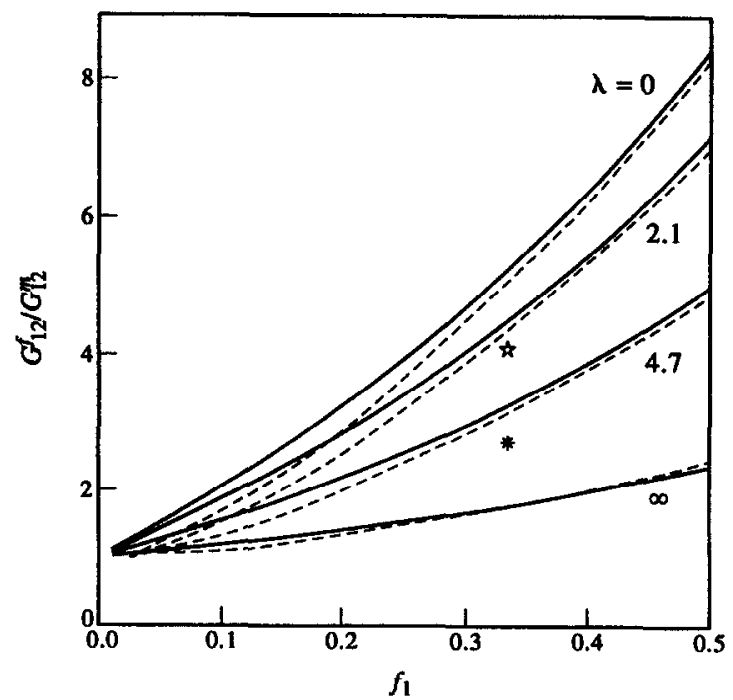

Fig. 4. Relative shear moduli $G_{12}$ of carbon-fiber-reinforced PPS as function of fiber volume fraction $f_{1}$. Symbols represent the experimental data. The theoretical curves are calculated by taking $t=16$ : solid line--present model; dashed line-laminated plate model.

the elastic moduli of a transversely isotropic composite can be explicitly expressed as follows:

$$
\mathbf{C}^{*}=\left[\begin{array}{cccccc}
C_{11}^{*} & C_{12}^{*} & C_{12}^{*} & 0 & 0 & 0 \\
C_{12}^{*} & C_{22}^{*} & C_{23}^{*} & 0 & 0 & 0 \\
C_{12}^{*} & C_{23}^{*} & C_{22}^{*} & 0 & 0 & 0 \\
0 & 0 & 0 & \frac{\left(C_{22}^{*}-C_{23}^{*}\right)}{2} & 0 & 0 \\
0 & 0 & 0 & 0 & C_{66}^{*} & 0 \\
0 & 0 & 0 & 0 & 0 & C_{66}^{*}
\end{array}\right],
$$

where

$$
\begin{aligned}
& C_{11}^{*}=\frac{4 X_{1}\left(3 K_{m}+4 \mu_{m}\right)-4\left(3 K_{m}-2 \mu_{m}\right) X_{2}}{3 r} \\
& C_{12}^{*}=\frac{4 X_{1}\left(3 K_{m}-2 \mu_{m}\right)-4 X_{2}\left(3 K_{m}+\mu_{m}\right)}{3 r} \\
& C_{22}^{*}=\frac{2 X_{4}\left(3 K_{m}+\mu_{m}\right)-2 X_{3}\left(3 K_{m}-2 \mu_{m}\right)}{3 r}+\frac{\mu_{m}}{2 X_{5}} \\
& C_{23}^{*}=\frac{2 X_{4}\left(3 K_{m}+\mu_{m}\right)-2 X_{3}\left(3 K_{m}-2 \mu_{m}\right)}{3 r}-\frac{\mu_{m}}{2 X_{5}} \\
& C_{66}^{*}=\frac{\mu_{m}}{2 X_{6}},
\end{aligned}
$$


where

$$
\begin{aligned}
& r=4\left(X_{1} X_{4}-X_{2} X_{3}\right) \\
& q=2\left[\left(f_{1} \bar{B}_{22}+f_{1} \bar{B}_{23}+1\right)\left(f_{1} \bar{B}_{11}+1\right)-2 f_{1}^{2} \bar{B}_{12} \bar{B}_{21}\right] \\
& X_{1}=\frac{f_{1}\left(f_{1} \bar{D}_{11}+1\right)\left(\bar{E}_{22}+\bar{E}_{23}\right) \cdot 2 f_{1}^{2} \bar{B}_{21} \bar{E}_{12}}{q}+\frac{1}{2} \\
& X_{2}=\frac{2 f_{1} \bar{E}_{12}\left(f_{1} \bar{B}_{22}+f \bar{B}_{23}+1\right)-2 f_{1}^{2}\left(\bar{E}_{22}+\bar{E}_{23}\right) \bar{B}_{12}}{q} \\
& X_{3}=\frac{2 f_{1} \bar{E}_{21}\left(f_{1} \bar{B}_{11}+1\right)-2 f_{1}^{2} \bar{E}_{11} \bar{B}_{21}}{q} \\
& X_{4}=\frac{2 f_{1} \bar{E}_{11}\left(f_{1} \bar{B}_{22}+f_{1} \bar{B}_{23}+1\right)-4 f_{1}^{2} \bar{E}_{21} \bar{B}_{12}}{q}+\frac{1}{2} \\
& X_{5}=\frac{f_{1} \bar{E}_{44}}{2 f_{1} \bar{B}_{44}+1}+\frac{1}{2} \\
& X_{6}=\frac{f_{1} \bar{E}_{66}}{2 f_{1} \bar{B}_{66}+1}+\frac{1}{2},
\end{aligned}
$$

where ( ) represents the volumetric average of ( ). Since $\bar{B}$ and $\bar{E}$ have a similar form, we can use $\bar{A}$ to represent $\bar{B}$ and $\bar{E}$. Its non-zero components can be written as follows:

$$
\begin{aligned}
\bar{A}_{11}= & \frac{1}{8}\left(A_{11}^{\prime}+A_{12}^{\prime}+4 A_{66}^{\prime}+A_{21}^{\prime}+5 A_{22}^{\prime}\right) b_{1}-\frac{1}{8}\left(A_{11}^{\prime}+A_{12}^{\prime}+4 A_{66}^{\prime}+A_{21}^{\prime}+5 A_{22}^{\prime}\right) b_{2} \\
& +\frac{3}{16}\left(3 A_{11}^{\prime}+A_{12}^{\prime}+4 A_{66}^{\prime}+A_{21}^{\prime}+5 A_{22}^{\prime}\right) b_{3}+\frac{1}{16}\left(3 A_{11}^{\prime}+A_{12}^{\prime}+4 A_{66}^{\prime}+A_{21}^{\prime}+5 A_{22}^{\prime}\right) b_{4} \\
& +\frac{5}{16}\left(A_{11}^{\prime}+A_{12}^{\prime}+4 A_{66}^{\prime}+A_{21}^{\prime}+A_{22}^{\prime}\right) b_{5}-\frac{1}{16}\left(A_{11}^{\prime}+A_{12}^{\prime}+4 A_{66}^{\prime}+A_{21}^{\prime}+A_{22}^{\prime}\right) b_{6} \\
\bar{A}_{12}= & \frac{1}{16}\left(A_{11}^{\prime}+3 A_{12}^{\prime}-4 A_{66}^{\prime}+5 A_{21}^{\prime}+A_{22}^{\prime}+6 A_{23}^{\prime}\right) b_{1} \\
& -\frac{1}{16}\left(A_{11}^{\prime}+3 A_{12}^{\prime}-4 A_{66}^{\prime}+5 A_{21}^{\prime}+A_{22}^{\prime}+6 A_{23}^{\prime}\right) b_{22} \\
& +\frac{3}{32}\left(A_{11}^{\prime}+7 A_{12}^{\prime}-4 A_{66}^{\prime}-5 A_{21}^{\prime}+A_{22}^{\prime}-4 A_{23}^{\prime}\right) b_{3} \\
& +\frac{1}{32}\left(A_{11}^{\prime}+7 A_{12}^{\prime}-4 A_{66}^{\prime}-5 A_{21}^{\prime}+A_{22}^{\prime}-4 A_{23}^{\prime}\right) b_{4} \\
& -\frac{5}{32}\left(A_{11}^{\prime}-A_{12}^{\prime}-4 A_{66}^{\prime}-A_{21}^{\prime}+A_{22}^{\prime}\right) b_{5}+\frac{1}{32}\left(A_{11}^{\prime}-A_{12}^{\prime}-4 A_{66}^{\prime}-A_{21}^{\prime}+A_{22}^{\prime}\right) b_{6} \\
\bar{A}_{23}= & \frac{1}{64}\left(5 A_{11}^{\prime}+19 A_{12}^{\prime}-20 A_{66}^{\prime}+19 A_{21}^{\prime}+5 A_{22}^{\prime}+16 A_{23}^{\prime}\right) b_{1} \\
& -\frac{1}{64}\left(5 A_{11}^{\prime}+19 A_{12}^{\prime}-20 A_{66}^{\prime}+19 A_{21}^{\prime}+5 A_{22}^{\prime}+16 A_{23}^{\prime}\right) b_{2} \\
& -\frac{3}{128}\left(5 A_{11}^{\prime}+11 A_{12}^{\prime}-20 A_{66}^{\prime}+11 A_{21}^{\prime}+5 A_{22}^{\prime}-32 A_{23}^{\prime}\right) b_{3} \\
& -\frac{1}{128}\left(5 A_{11}^{\prime}+11 A_{12}^{\prime}-20 A_{66}^{\prime}+11 A_{21}^{\prime}+5 A_{22}^{\prime}-32 A_{23}^{\prime}\right) b_{4} \\
& +\frac{5}{128}\left(A_{11}^{\prime}-A_{12}^{\prime}-4 A_{66}^{\prime}-A_{21}^{\prime}+A_{22}^{\prime}\right) b_{5}-\frac{1}{128}\left(A_{11}^{\prime}-A_{12}^{\prime}-4 A_{66}^{\prime}-A_{21}^{\prime}+A_{22}^{\prime}\right) b_{6} \\
\bar{A}_{32}= & \bar{A}_{23} \\
\bar{A}_{44}= & \frac{1}{2}\left(\bar{A}_{22}-\bar{A}_{23}\right) \\
\bar{A}_{55}= & \frac{1}{16}\left(A_{11}^{\prime}-A_{12}^{\prime}+6 A_{66}^{\prime}-A_{21}^{\prime}+4 A_{22}^{\prime}-3 A_{23}^{\prime}\right) b_{1} \\
& -\frac{1}{16}\left(A_{11}^{\prime}-A_{12}^{\prime}+6 A_{66}^{\prime}-A_{21}^{\prime}+4 A_{22}^{\prime}-3 A_{23}^{\prime}\right) b_{2} \\
& +\frac{3}{23}\left(A_{11}^{\prime}-A_{12}^{\prime}-A_{21}^{\prime}+A_{22}^{\prime}-2 A_{23}^{\prime}\right) b_{3}+\frac{1}{32}\left(A_{11}^{\prime}-A_{12}^{\prime}-A_{21}^{\prime}+A_{22}^{\prime}-2 A_{23}^{\prime}\right) b_{4} \\
& -\frac{5}{32}\left(A_{11}^{\prime}-A_{12}^{\prime}-4 A_{66}^{\prime}-A_{21}^{\prime}+A_{22}^{\prime}\right) b_{5}+\frac{1}{32}\left(A_{11}^{\prime}-A_{12}^{\prime}-4 A_{66}^{\prime}-A_{21}^{\prime}+A_{22}^{\prime}\right) b_{6} \\
&
\end{aligned}
$$




$$
\begin{aligned}
\bar{A}_{66}= & \bar{A}_{55} \\
\bar{A}_{13}= & \bar{A}_{12} \\
\bar{A}_{21}= & \frac{1}{16}\left(A_{11}^{\prime}+3 A_{21}^{\prime}-4 A_{66}^{\prime}+5 A_{12}^{\prime}+A_{22}^{\prime}+6 A_{23}^{\prime}\right) b_{1} \\
& -\frac{1}{16}\left(A_{11}^{\prime}+3 A_{21}^{\prime}-4 A_{66}^{\prime}+5 A_{12}^{\prime}+A_{22}^{\prime}+6 A_{23}^{\prime}\right) b_{2} \\
& +\frac{3}{32}\left(A_{11}^{\prime}+7 A_{21}^{\prime}-4 A_{66}^{\prime}-5 A_{12}^{\prime}+A_{22}^{\prime}-4 A_{23}^{\prime}\right) b_{3} \\
& +\frac{1}{32}\left(A_{11}^{\prime}+7 A_{21}^{\prime}-4 A_{66}^{\prime}-5 A_{12}^{\prime}+A_{22}^{\prime}-4 A_{23}^{\prime}\right) b_{4} \\
& -\frac{5}{32}\left(A_{11}^{\prime}-A_{21}^{\prime}-4 A_{66}^{\prime}-A_{12}^{\prime}+A_{22}^{\prime}\right) b_{5}+\frac{1}{32}\left(A_{11}^{\prime}-A_{21}^{\prime}-4 A_{66}^{\prime}-A_{12}^{\prime}+A_{22}^{\prime}\right) b_{6} \\
\bar{A}_{31}= & \bar{A}_{21} \\
\bar{A}_{22}= & \frac{1}{64}\left(15 A_{11}^{\prime}+9 A_{12}^{\prime}+36 A_{66}^{\prime}+9 A_{21}^{\prime}+31 A_{22}^{\prime}\right) b_{1} \\
& -\frac{1}{64}\left(15 A_{11}^{\prime}+9 A_{12}^{\prime}+36 A_{66}^{\prime}+9 A_{21}^{\prime}+31 A_{22}^{\prime}\right) b_{2} \\
& -\frac{3}{128}\left(15 A_{11}^{\prime}+A_{12}^{\prime}+4 A_{66}^{\prime}+A_{21}^{\prime}-17 A_{22}^{\prime}\right) b_{3} \\
& -\frac{1}{128}\left(15 A_{11}^{\prime}+A_{12}^{\prime}+4 A_{66}^{\prime}+A_{21}^{\prime}-17 A_{22}^{\prime}\right) b_{4} \\
& +\frac{15}{128}\left(A_{11}^{\prime}-A_{12}^{\prime}-4 A_{66}^{\prime}-A_{21}^{\prime}+A_{22}^{\prime}\right) b_{5}-\frac{3}{128}\left(A_{11}^{\prime}-A_{12}^{\prime}-4 A_{66}^{\prime}-A_{21}^{\prime}+A_{22}^{\prime}\right) b_{6} \\
\bar{A}_{33}= & \bar{A}_{22},
\end{aligned}
$$

where

$$
\begin{aligned}
& b_{1}=\frac{\exp \left[\frac{\pi}{2} \lambda\right]}{\exp \left[\frac{\pi}{2} \lambda\right]-\lambda} \\
& b_{2}=\frac{\lambda}{\exp \left[\frac{\pi}{2} \lambda\right]-\lambda} \\
& b_{3}=\frac{\exp \left[\frac{\pi}{2} \lambda\right]}{\exp \left[\frac{\pi}{2} \lambda\right]-\lambda} \frac{1+\lambda^{2}}{9+\lambda^{2}} \\
& b_{4}=\frac{\lambda}{\exp \left[\frac{\pi}{2} \lambda\right]-\lambda} \frac{1+\lambda^{2}}{9+\lambda^{2}} \\
& b_{5}=\frac{\exp \left[\frac{\pi}{2} \lambda\right]}{\exp \left[\frac{\pi}{2} \lambda\right]-\lambda} \frac{1+\lambda^{2}}{25+\lambda^{2}} \\
& b_{6}=\frac{\lambda}{\exp \left[\frac{\pi}{2} \lambda\right]-\lambda} \frac{1+\lambda^{2}}{25+\lambda^{2}} .
\end{aligned}
$$

When $\lambda \rightarrow 0$ and $\lambda \rightarrow \infty, b_{1}, b_{2}, b_{3}, b_{4}, b_{5}$ and $b_{6}$ can be represented as: 


$$
\begin{aligned}
& \lim _{i \rightarrow 0} b_{1}=1, \quad \lim _{i \rightarrow 0} b_{2}=0, \quad \lim _{i \rightarrow 0} b_{3}=\frac{1}{9}, \quad \lim _{\lambda \rightarrow 0} b_{4}=0, \\
& \lim _{\lambda \rightarrow 0} h_{5}=\frac{1}{25}, \quad \lim _{2,0} b_{6}=0 ; \quad \lim _{2 \ldots} b_{1}=1, \quad \lim _{\lambda \ldots \infty} b_{2}=0, \\
& \lim _{i \rightarrow x_{0}} b_{3}=1, \lim _{\lambda \rightarrow \infty} b_{4}=0, \lim _{i \rightarrow x} b_{5}=1, \lim _{\lambda \rightarrow \infty} b_{6}=0 .
\end{aligned}
$$

When $\lambda \rightarrow 0$, substituting eqn (31) into eqn (29) leads to

$$
\begin{aligned}
& \bar{A}_{11}=\bar{A}_{22}=\bar{A}_{33}=\frac{1}{15}\left(3 A_{11}^{\prime}+2 A_{12}^{\prime}+8 A_{66}^{\prime}+2 A_{21}^{\prime}+8 A_{22}^{\prime}\right) \\
& \bar{A}_{44}=\bar{A}_{55}=\bar{A}_{66}=\frac{1}{30}\left(2 A_{11}^{\prime}-2 A_{12}^{\prime}+12 A_{66}^{\prime}-2 A_{21}^{\prime}+7 A_{22}^{\prime}-5 A_{33}^{\prime}\right) \\
& \bar{A}_{12}=\bar{A}_{21}=\bar{A}_{23}=\frac{1}{15}\left(A_{11}^{\prime}+4 A_{12}^{\prime}-4 A_{66}^{\prime}+4 A_{21}^{\prime}-A_{23}^{\prime}+5 A_{23}^{\prime}\right) .
\end{aligned}
$$

From eqn (32) we know that when $\lambda \rightarrow 0$, the short fibers are randomly distributed in the matrix. Therefore, $\bar{A}$ is an isotropic tensor.

When $\lambda \rightarrow \infty$, substituting eqn (31) into eqn (29) leads to

$$
\bar{A}=A^{\prime} .
$$

From eqn (33), we know that when $\lambda \rightarrow \infty$, the short fibers are perfectly aligned in the matrix. The non-zero components of the $\underset{\sim}{B^{\prime}}$ and $\underset{\sim}{E^{\prime}}$ can be written as follows :

$$
\begin{gathered}
B_{11}^{\prime}=\frac{4 a_{1}-2\left(S_{22}+S_{23}\right)}{p} \\
B_{21}^{\prime}=B_{31}^{\prime}=\frac{-2\left(a_{2}-S_{21}\right)}{p} \\
B_{12}^{\prime}=B_{13}^{\prime}=\frac{-2\left(a_{3}-S_{12}\right)}{p} \\
B_{22}^{\prime}=B_{33}^{\prime}=\frac{a_{4}-S_{11}}{p}+\frac{1}{4\left(a_{5}-S_{44}\right)} \\
B_{23}^{\prime}=B_{32}^{\prime}=\frac{a_{4}-S_{11}}{p}-\frac{1}{4\left(a_{5}-S_{44}\right)} \\
B_{55}^{\prime}=B_{66}^{\prime}=\frac{1}{4\left(a_{6}-S_{66}\right)} \\
B_{44}^{\prime}=\frac{1}{4\left(a_{6}-S_{44}\right)} \\
E_{23}^{\prime}=E_{32}^{\prime}=\frac{1}{p}\left[\left(S_{22}+S_{23}-1\right)\left(a_{4}-S_{11}\right)-S_{21}\left(a_{3}-S_{12}\right)\right]-\frac{2 S_{44}-1}{4\left(a_{5}-S_{44}\right)} \\
E_{21}^{\prime}=E_{31}^{\prime}=\frac{1}{p}\left[\left(S_{11}-1\right)\left(4 a_{11}-2 S_{22}-2 S_{22}-2 S_{23}\right)-4 S_{12}\left(a_{2}-S_{21}\right)\right] \\
E_{12}^{\prime}=E_{13}^{\prime}=\frac{1}{p}\left[2 S_{12}\left(a_{4}-S_{11}\right)-2\left(S_{11}-1\right)\left(a_{3} \cdot S_{12}\right) \cdot\right] \\
\frac{1}{p}\left[\left(S_{22}+S_{23}-1\right)\left(a_{4}-S_{11}\right)-S_{21}\left(a_{3}-S_{12}\right)\right]+\frac{2 S_{44}-1}{4\left(a_{5}-S_{44}\right)} \\
\left.\left.E_{23}\right)-2\left(S_{22}+S_{23}-1\right)\left(a_{2}-S_{21}\right)\right]
\end{gathered}
$$




$$
\begin{aligned}
& E_{66}^{\prime}=E_{55}^{\prime}=\frac{2 S_{66}-1}{4\left(a_{6}-S_{66}\right)} \\
& E_{44}^{\prime}=\frac{2 S_{44}-1}{4\left(a_{5}-S_{44}\right)}
\end{aligned}
$$

where

$$
\begin{gathered}
a_{1}=\frac{K_{m}}{3\left(K_{m}-K_{f}\right)}+\frac{1}{6} \frac{\mu_{m}}{\mu_{m}-\mu_{f}} \\
a_{3}=a_{2}=\frac{K_{m}}{3\left(K_{m}-K_{f}\right)}-\frac{1}{3} \frac{\mu_{m}}{\mu_{m}-\mu_{f}} \\
a_{4}=\frac{K_{m}}{3\left(K_{m}-K_{f}\right)}+\frac{2}{3} \frac{\mu_{m}}{\mu_{m}-\mu_{f}} \\
a_{5}=a_{6}=\frac{\mu_{m}}{2\left(\mu_{m}-\mu_{f}\right)} \\
p=2\left[\left(2 a_{1}-S_{22}-S_{23}\right)\left(a_{4}-S_{11}\right)-2\left(a_{2}-S_{21}\right)\left(a_{3}-S_{12}\right)\right] .
\end{gathered}
$$

4.3.2. Numerical results. It is of interest to see how (i) the fiber distribution, when it changes from the uniaxial directional $(\lambda=\infty)$ to random distribution $(\lambda=0)$, (ii) the volume fraction, $f_{1}$, and (iii) the aspect ratio, $t$, of the reinforced fibers affect the five elastic constants of a transversely isotropic composite. To this end, we used the properties of glass fibers and the polyphenylene sulfide (PPS) matrix in our calculations. The elastic constants are

PPS :

$$
E_{11}^{m}=4 \mathrm{GPa}, \quad v_{12}^{m}=0.4
$$

Glass fibers :

$$
E_{11}^{f_{1}}=76 \mathrm{GPa}, \quad v_{12}^{f_{2}}=0.25 \text {. }
$$

In reading these results, we are reminded once again that direction 1 is the infinitely extended direction, direction 2 is perpendicular to the surface of the thickness, and direction 3 is along the width. Compared with the tensile and shear moduli of $E_{11}^{m}=4 \mathrm{GPa}$ and $G_{12}^{m}=1.43 \mathrm{GPa}$ for the PPS matrix, the elastic moduli of the composites are significantly higher, clearly revealing the reinforcing effect of the fibers. To gain deeper understanding of the effects of various parameters, we have calculated and plotted the elastic moduli of $f_{1}=30 \%$ glass-fiber-reinforced PPS as functions of the aspect ratio, $t$, in Figs 5(a-e). They show that all the moduli for randomly distributed glass-fiber reinforced composites ( $\lambda$ is small) increase with increasing $t$. The elastic moduli, $E_{11}, E_{22}, G_{12}$, increase with increasing $t$ for all $\lambda$, but for $v_{12}$ and $G_{23}$ they decrease with increasing $t$. The effect becomes saturated above $t=20$. These results emphasize the importance of choosing a suitable processing technique to increase the fiber aspect ratio, $t$.

Next we turn our attention to the fiber volume fraction, $f_{1}$, dependence of elastic moduli as a function of $\lambda$. The values of $f_{1}=0.1,0.2,0.3$ and 0.4 were chosen for demonstration. The five elastic constants of a transversely isotropic composite as a function of $\lambda$ are depicted in Figs $6(\mathrm{a}-\mathrm{e})$. The elastic modulus, $E_{11}$, increases with increasing $\lambda$, but $E_{22}$ and $G_{23}$ decrease with increasing $\lambda$. This shows that the more oriented the fibers are in one direction ( $\lambda$ increases) the greater is the reinforcing effect in that direction, but vice versa in another direction. The reinforcing effects of the elastic moduli $G_{12}$ and $v_{12}$ both have peak values at $\lambda=2.5$ for $G_{12}$ and $\lambda=5.0$ for $v_{12}$. 
(a)

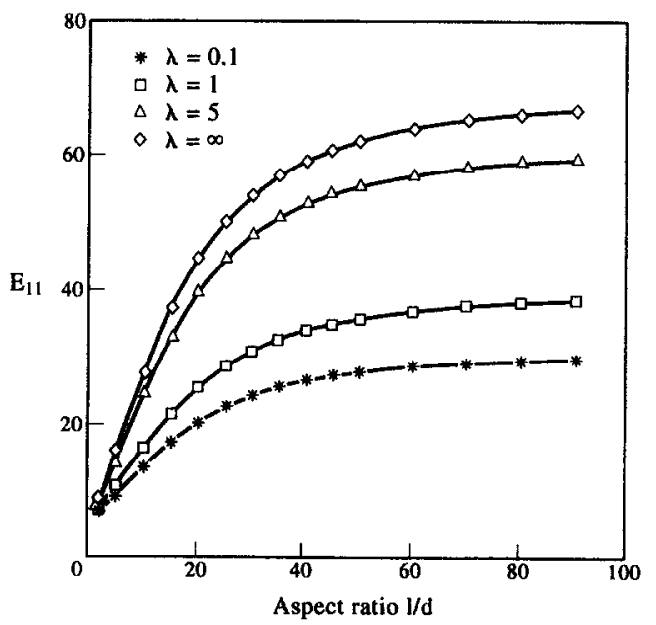

(c)

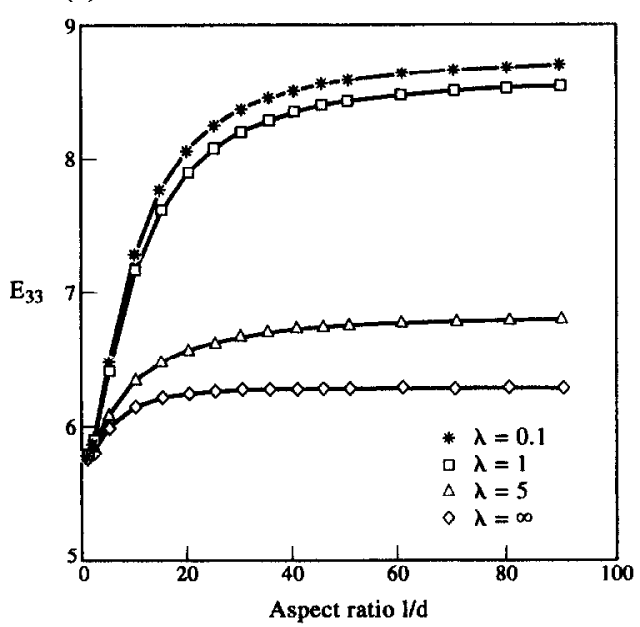

(e)

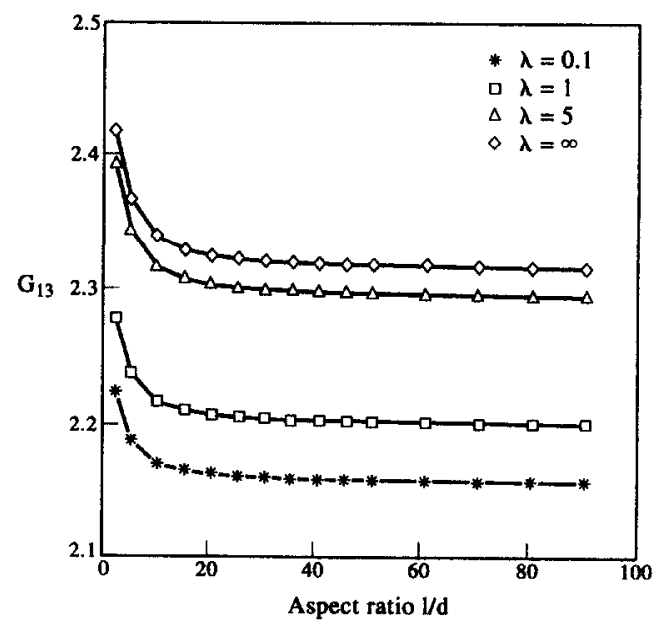

(b)

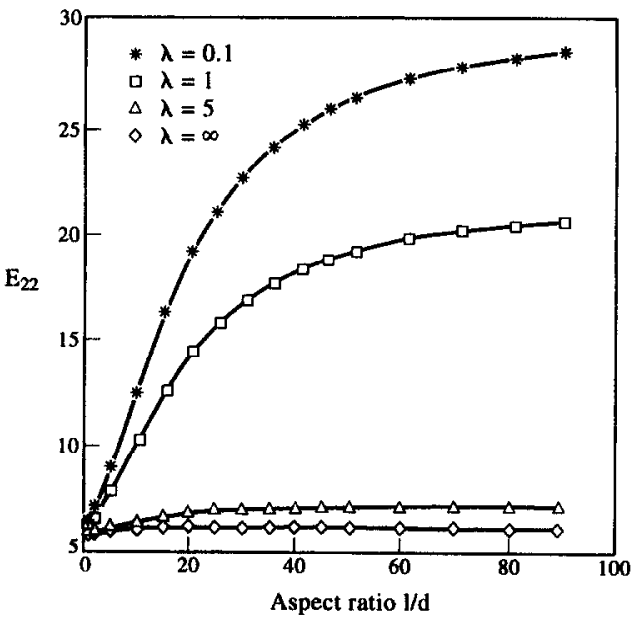

(d)

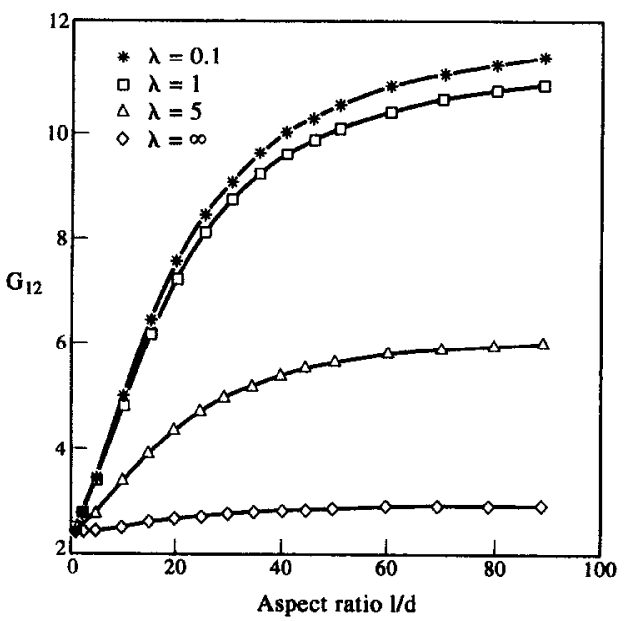

(f)

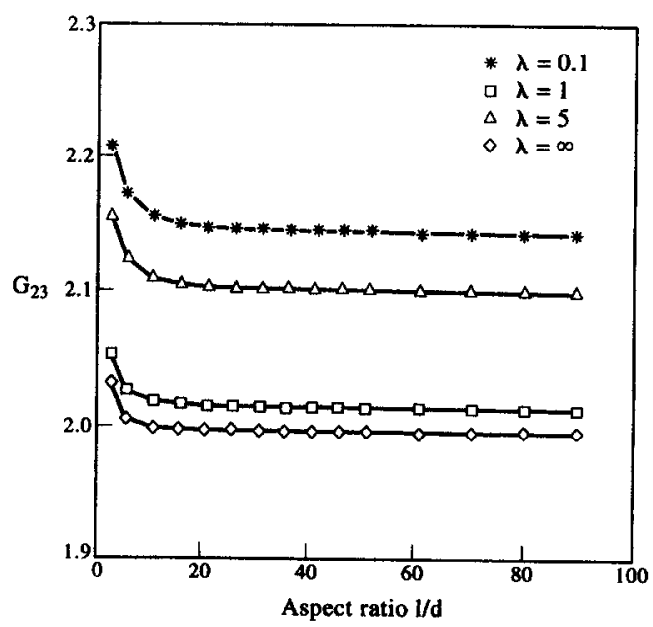


(g)

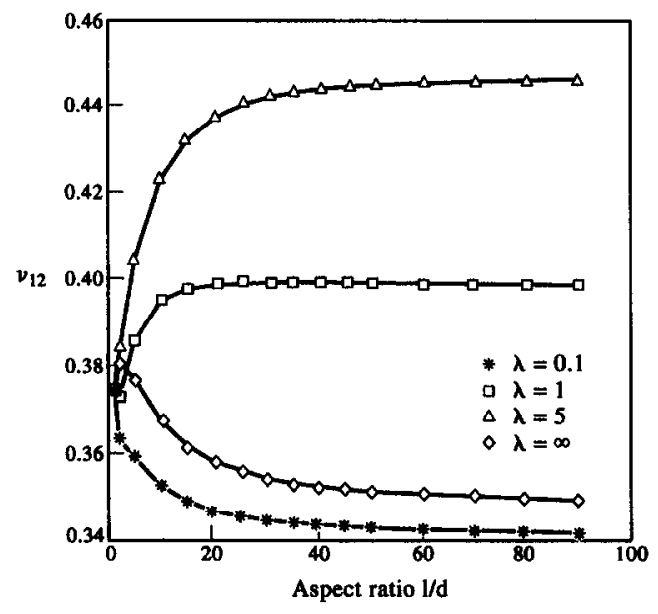

(i) (h)

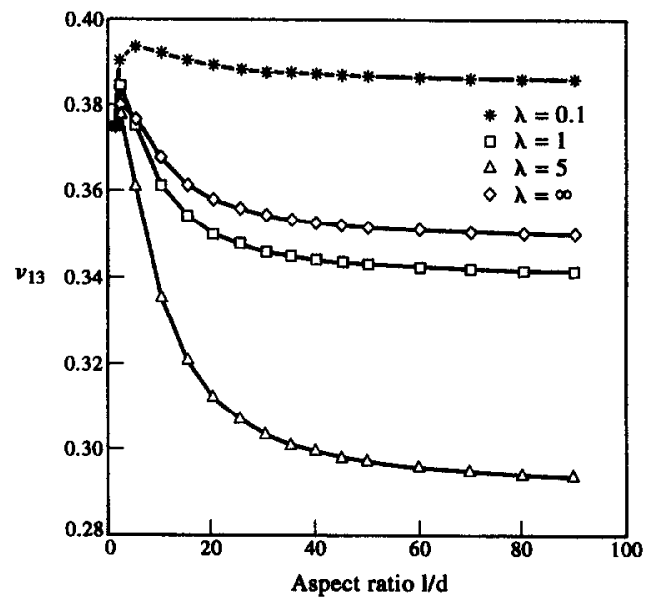

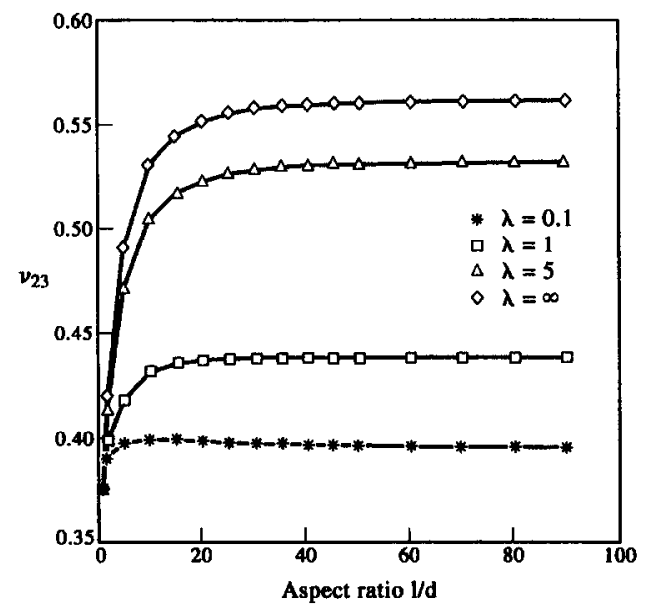

Fig. 5. The fiber orientation factor $\lambda$ dependence of the elastic moduli as functions of aspect ratio, $t$, at $f_{1}=30 \%:$ : (a) $E_{11}$, (b) $E_{22}$, (c) $G_{12}$, (d) $G_{23}$, (e) $v_{12}$. 
(a)

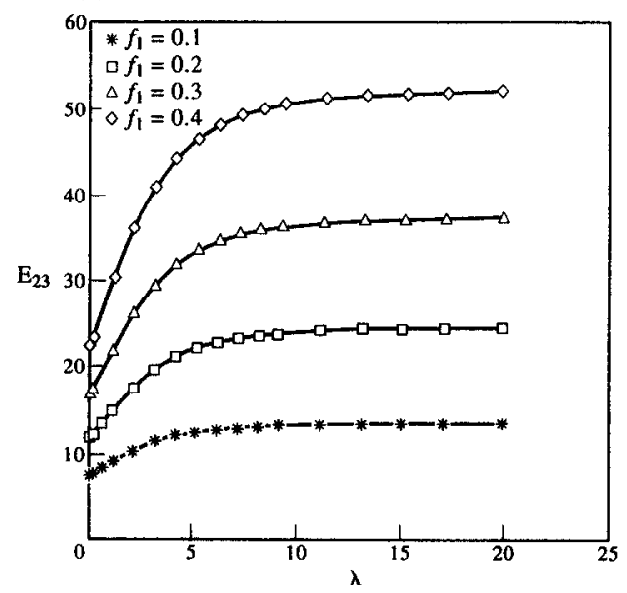

(c)

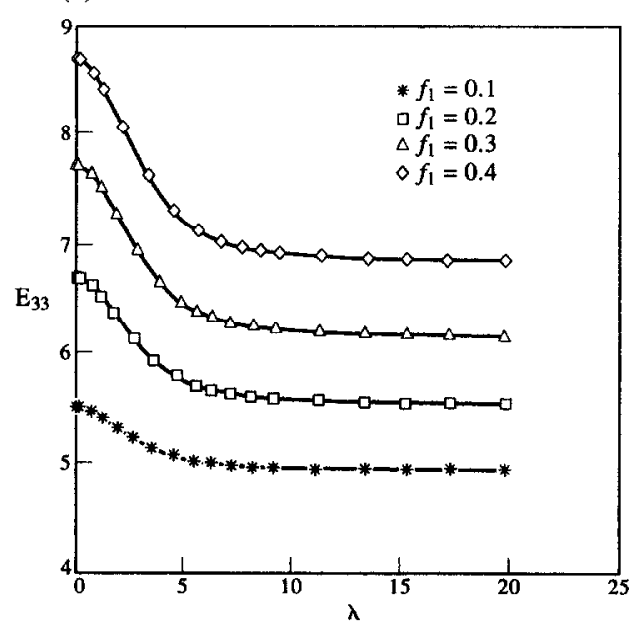

(e)

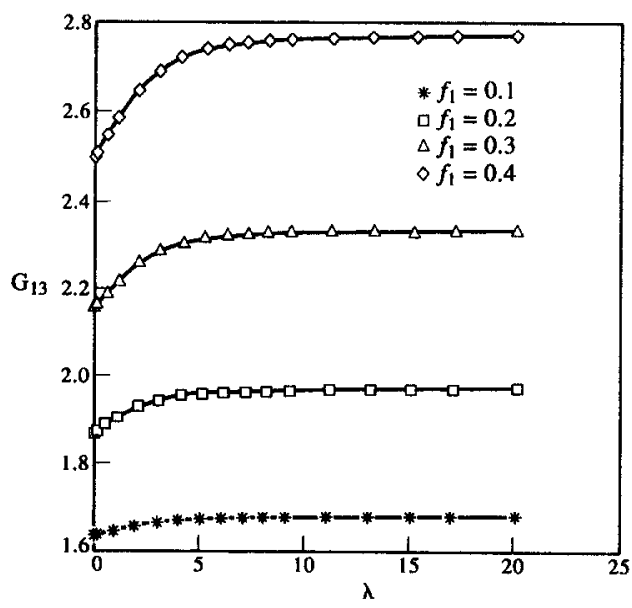

(b)

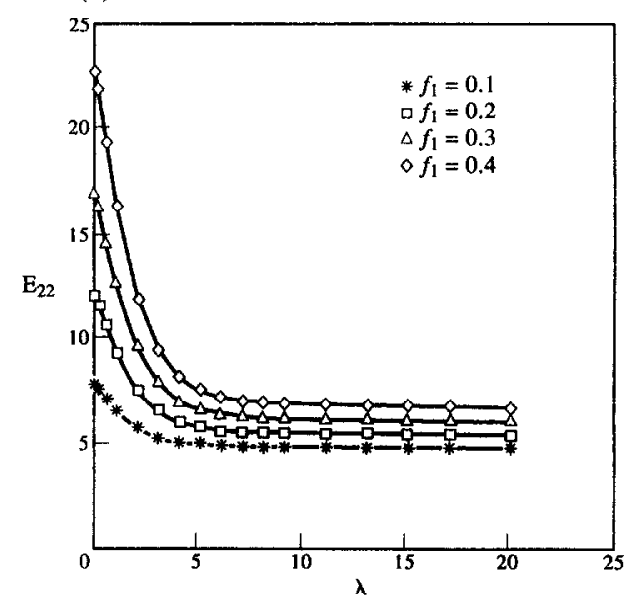

(d)

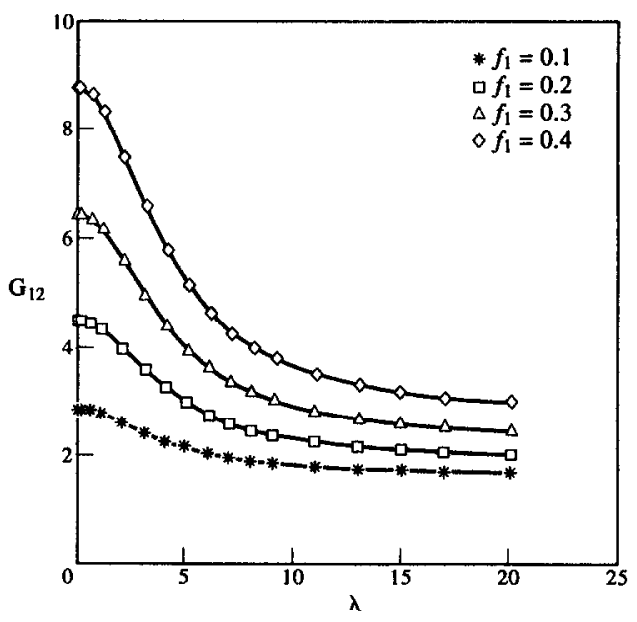

(f)

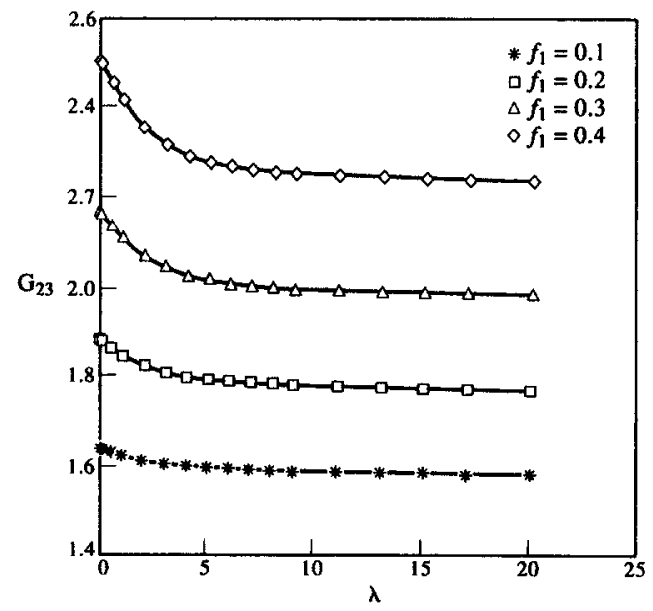


(g)

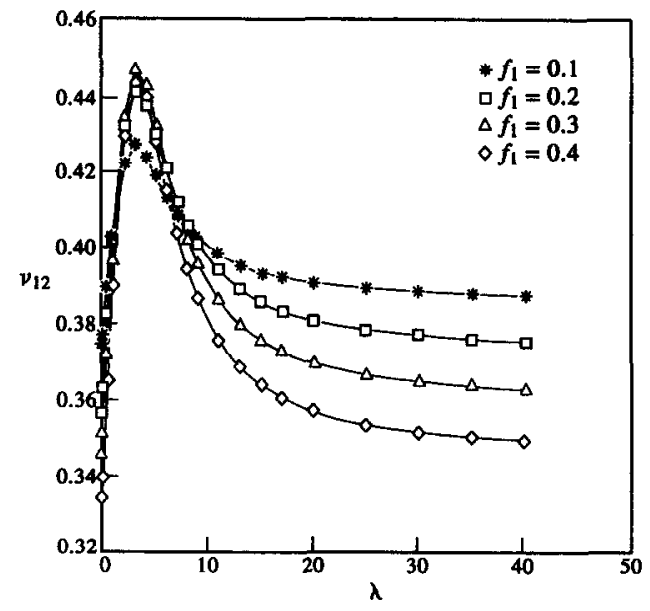

(h)

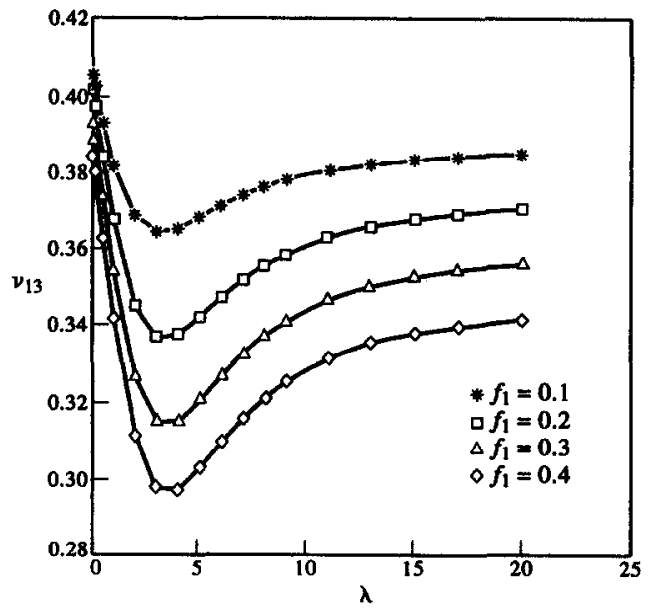

(i)

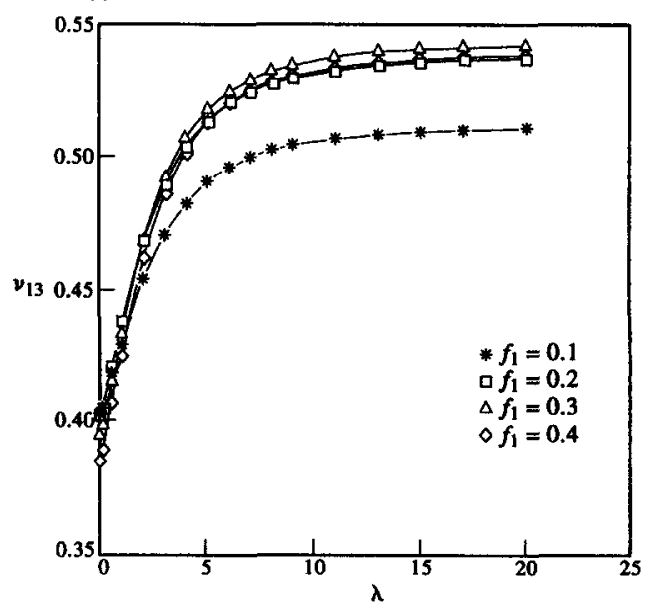

Fig. 6. The volume fractions dependence of the elastic moduli as functions of fiber orientation factor, $\lambda$, at $t=15:$ (a) $E_{11}$, (b) $E_{22}$, (c) $G_{12}$, (d) $G_{23}$, (e) $v_{13}$.

\section{CONCLUSION}

The elastic moduli of a short-fiber-reinforced composite depend on the corresponding properties of the fiber and matrix, and the volume fraction, $f_{1}$, aspect ratio, $t$, and orientation factor, $\lambda$, of the fibers. Since the elastic moduli of high-performance polymers commonly used as matrices are quite similar, the choice of polymer matrix is not an important factor in the enhancement of stiffness. An increase in the fiber volume fraction leads to increases in all the elastic moduli, with $E_{11}$ increasing faster than linearly with $f_{1}$. The remaining two factors affecting the elastic moduli may be controlled by processing techniques. We have seen that all the moduli for glass-fiber composites increase with increasing $t$, but that the increase is much more significant for the longitudinal tensile modulus $E_{11}$. Therefore, it is imperative to optimize the conditions for compounding and processing of composites to give fibers high aspect ratios, preferably with $t>20$.

Acknowledgement-This work was supportcd by the National Science Council of P. R. China under grant NSC83-0401-E002-145.

\section{REFERENCES}

Chou, T. W. and Nomura, S. (1980). Fiber urientation effects on the thermoelastic properties of short-fiber composites. Fiber Sci. Technol. 14, 279-291.

Chou, T. W., Nomura, S. and Taya, M. (1980). A self-consistent approach to the elastic stiffness of a short-fiber composite. J. Compos. Mater. 14, 178-188. 
Choy, C. L., Leung, W. P., Kowk, K. W. and Lau Felix, P. (1992). Elastic moduli and thermal conductivity of injection-molded short-fiber-reinforced thermoplastics. Polymer Compos. 13, 6980.

Christensen, R. M. (1979). Mechanics of Composite Materials. Wiley-Interscience, New York.

Eshelby, J. D. (1957). The determination of the elastic field of an ellipsoidal inclusion and related problems. Proc. R. Soc. Lon. A241, 376-396.

Hahn, H. T., Jernia, K. L. and Chiou, W. (1986) Proc. Int. Symp. on Composite Materials and Structures, Beijing, China, p. 68.

Kacir, L., Narkis, M. and 1shai, O. (1975). Oriented short glass-fiber composites: I. Preparation and statistical analysis of aligned fiber materials. Polymer Engng Sci. 15, 525-531.

Mori, T. and Tanaka, K. (1973). Average stress in matrix and average elastic energy of materials with misfitting inclusions. Acta Mettall. 21, 571-574.

Mura, T. (1982) Micromechanics of Defects in Solids. Martinus Nijhoff, Dordrecht, The Netherlands.

Takao, Y., Chou, T. W. and Taya, M. (1982). Effective longitudinal Young's modulus of misoriented short fiber composites. ASME J. Appl. Mech. 49, 536-540.

Taya, M. and Chou, T. W. (1981). On two kinds of ellipsoidal inhomogeneities in an infinite elastic body: an application to a hybrid composite. Int. J. Solids Structures 17, 553-563.

Taya, M. and Mura, T. (1981). On stiffness and strength of an aligned short fiber reinforced composites containing fiber end cracks under uniaxial applied stress. ASME J. Appl. Mech. 48, 361-367.

Tsai, S. W. and Hahan, H. T. (1980). Introduction to Composite Materials. Technomic, CT, USA.

\section{APPENDIX}

Components of Eshelby's $\mathrm{S}_{\mathrm{ijk}}$ tensor

For a spheroidal inclusion aligned along axis 1, the components of Eshelby's tensor $S_{i j k}$ are given by

$$
\begin{aligned}
& S_{1111}=\frac{1}{2\left(1-v_{0}\right)}\left[1-2 v_{10}+\frac{3 t^{2}-1}{t^{2}-1}-\left(1-2 v_{10}+\frac{3 t^{2}}{t^{2}-1}\right) g\right] \\
& S_{2222}=S_{3333}=\frac{3}{8\left(1-v_{0}\right)} \frac{t^{2}}{\left(t^{2}-1\right)}+\frac{1}{4\left(1-v_{11}\right)}\left[1-2 v_{0}-\frac{9}{4\left(t^{2}-1\right)}\right] g \\
& S_{2233}=S_{3,322}=\frac{1}{4\left(1-v_{0}\right)}\left\{\frac{t^{2}}{2\left(t^{2}-1\right)}-\left[1-2 v_{0}+\frac{3}{4\left(t^{2}-1\right)}\right] g\right\} \\
& S_{2211}=S_{3311}=-\frac{1}{2\left(1-v_{0}\right)} \frac{t^{2}}{\left(t^{2}-1\right)}+\frac{1}{4\left(1-v_{0}\right)}\left(\frac{3 t^{2}}{t^{2}-1}-1+2 v_{0}\right) g \\
& S_{1122}=S_{1133}=\frac{1}{2\left(1 \cdots v_{0}\right)}\left\{2 v_{0}-1-\frac{1}{t^{2}-1}+\left[1-2 v_{11}+\frac{3}{2\left(r^{2}-1\right)}\right] g\right\} \\
& S_{2,3,3,3}=\frac{1}{4\left(1-v_{01}\right)}\left\{\frac{t^{2}}{2\left(t^{2}-1\right)}+\left[1-2 v_{11}-\frac{3}{4\left(t^{2}-1\right)}\right] g\right\} \\
& S_{1212}=S_{1313}=\frac{1}{4\left(1-v_{0}\right)}\left\{1-2 v_{0}-\frac{t^{2}+1}{t^{2}-1}-\frac{1}{2}\left[1-2 v_{0}-\frac{3 t^{2}+3}{t^{2}-1}\right] g\right\} \text {, }
\end{aligned}
$$

where $v_{0}$ is the Poisson's ratio of the matrix, $t$ is the ratio of the inclusion $(=l / d)$ and $g$ is given by

$$
\begin{aligned}
& g=\frac{t}{\left(t^{2}-1\right)^{3 / 2}}\left[t\left(t^{2}-1\right)^{1 / 2}-\cosh ^{1} t\right] \text { for prolate inclusions } \\
& g=\frac{t}{\left(1-t^{2}\right)^{3 / 2}}\left[\cos ^{-1} t-t\left(1-t^{2}\right)^{1 / 2}\right] \text { for oblateinclusions } \\
& g-2 / 3 \text { for spheres. }
\end{aligned}
$$

For spherical inclusion, they can be simplified to

$$
\begin{aligned}
& S_{1111}=S_{2222}=S_{1333}=\frac{7-5 v_{0}}{15\left(1-v_{0}\right)} \\
& S_{1122}=S_{2233}=S_{3311}=\frac{5 v_{0}-1}{15\left(1-v_{0}\right)} \\
& S_{1212}=S_{2323}=S_{3131}=\frac{4-5 v_{0}}{15\left(1-v_{0}\right)} .
\end{aligned}
$$

For a circular cylinder, one has 
Effective elastic moduli of fiber composites

$$
\begin{aligned}
& S_{1111}=0 \\
& S_{2222}=S_{3333}=\frac{5-4 v_{0}}{8\left(1-v_{0}\right)} \\
& S_{2233}=S_{3311}=\frac{4 v_{0}-1}{8\left(1-v_{0}\right)} \\
& S_{2211}=S_{3311}=\frac{v_{0}}{2\left(1-v_{0}\right)} \\
& S_{1122}=S_{1133}=0 \\
& S_{2323}=\frac{3-4 v_{0}}{8\left(1-v_{0}\right)} \\
& S_{1212}=S_{1313}=\frac{1}{4} .
\end{aligned}
$$

For a thin disc with $t \rightarrow 0$, the non-vanishing components are

$$
\begin{aligned}
& S_{1111}=1 \\
& S_{1122}=S_{1133}=\frac{v_{0}}{1-v_{0}} \\
& S_{1212}=S_{1313}=\frac{1}{2} .
\end{aligned}
$$

